$\left(\mathrm{FEV}_{1}\right)$, HDL-cholesterol (HDL), body mass index (BMI), systolic blood pressure (BP), and glycated haemoglobin (HbA1c). In men, those in stable manual and upward (vs stable non-manual) class categories showed higher BMI, HbA1c and BP, and lower HDL and $\mathrm{FEV}_{1} \quad(\mathrm{p}<0.10)$. Limited development was associated with higher BMI and $\mathrm{HbA1c}$, and lower $\mathrm{FEV}_{1}(\mathrm{p}<0.05)$. In women, those in the stable manual class had lower HDL and $\mathrm{FEV}_{1}$, and higher HbA1c $(p<0.05)$. Downward mobility was associated with lower HDL $(p=0.018)$. Compensatory development was associated with higher $\mathrm{FEV}_{1}(\mathrm{p}<0.05)$. In both sexes, the socioeconomic- and developmental plasticity- biomarker associations remained significant in the combined analysis. Socioeconomic circumstances and developmental plasticity were associated with negative biomarker outcomes, although they follow independent associative pathways.

\section{P1-307 HIGHER CANCER DETECTION RISK IN WOMEN WITH A FALSE POSITIVE RESULT IN BREAST CANCER SCREENING IN SPAIN}

doi:10.1136/jech.2011.142976e.99

\begin{abstract}
${ }^{1} \mathrm{~J}$ Blanch, ${ }^{1} \mathrm{~A}$ Romero, ${ }^{1} \mathrm{M}$ Sala, ${ }^{1} \mathrm{R}$ Román, ${ }^{*}{ }^{2} \mathrm{D}$ Salas, ${ }^{3} \mathrm{R}$ Zubizarreta, ${ }^{4} \mathrm{M}$ Ederra, ${ }^{5} \mathrm{M}$ Baré, ${ }^{6} \mathrm{~J}$ Galceran, ${ }^{7} \mathrm{I}$ González-Román, ${ }^{8} \mathrm{C}$ Natal, ${ }^{9} \mathrm{M}$ de la Vega, ${ }^{10} \mathrm{~A}$ Baroja, ${ }^{1} \mathrm{~F}$ Macià, ${ }^{1} \mathrm{X}$ Castells. ${ }^{1}$ Department of Epidemiology and Evaluation, IMIM-Parc de Salut Mar, CIBERESP, Barcelona, Spain; ${ }^{2}$ General Directorate Public Health \& Centre for Public Health Research, Valencia, Spain; ${ }^{3}$ Galician breast cancer screening programme. Public health \& Planning Directorate. Health Office, Santiago de Compostela, Spain; ${ }^{4}$ Navarra Breast Cancer Screening Programme. Public Health Institute, CIBERESP, Pamplona, Spain; ${ }^{5}$ OTC-Epidemiology, Sabadell, Spain; ${ }^{6}$ PDPCM, League Foundation and Cancer Prevention Research, IISPV, Reus, Spain; ${ }^{7}$ Castilla-Leon Breast Cancer Screening Programme, D G Salud Pública ID e I, SACYL, Valladolid, Spain; ${ }^{8}$ Unit of Analysis and Programs. Health Service of Asturias, Oviedo, Spain; ${ }^{9}$ General Directorate of Health Care Programmes. Canary Islands Health Service, Santa Cruz de Tenerife, Spain; ${ }^{10}$ La Rioja Breast Cancer Screening Programme, Fundacion Rioja Salud, Logroño, Spain
\end{abstract}

Introduction Breast screening reduces mortality from breast cancer through early detection. Despite its benefits, breast cancer screening presents some adverse effects, as false positive (FP) results. Our aim was to estimate the risk of cancer detection associated to have experienced a previous FP mammography.

Methods The Cumulative False Positive Risk study includes eight population-based screening programs in Spain, between 1990 and 2006, that invites women aged between $45 / 50$ and 69 years, with no previous breast cancer. We included women participating in at least two screenings rounds. A positive mammogram reading was considered a FP result if, after further assessments, breast cancer was not diagnosed. Cancer detection risk was estimated through $\mathrm{OR}$ with a multivariate discrete-time-hazard model with a random intercept. The model included adjusting variables related to screening programs (radiologic unit, reading method and number of projections) and woman (age, hormone replacement therapy use, menopausal status, previous invasive procedures and familial history of breast cancer).

Results Women who had experienced at least one previous FP result had a higher cancer detection rate than those without a FP (4.72 per 1000 mammograms vs 2.56 per 1000 mammograms). The adjusted $\mathrm{OR}$ of cancer detection was higher in women with a FP in any previous screening $(\mathrm{OR}=1.8895 \% \mathrm{CI} 1.76$ to 2.00$)$.
Conclusion Women with a previous FP result had a higher risk of cancer detection. This result may suggest that factors related to FP could provide useful information to redesign different early detection strategies for specific subgroups of women.

\section{P1-308 EFFECT OF FALSE-POSITIVES AND WOMEN'S CHARACTERISTICS ON THE LONG-TERM ATTITUDE TOWARDS BREAST CANCER SCREENING}

doi:10.1136/jech.2011.142976e.100

${ }^{1} \mathrm{R}$ Román, ${ }^{* 1} \mathrm{M}$ Sala, ${ }^{2} \mathrm{R}$ Zubuzarreta, ${ }^{3}$ Delfrade, ${ }^{4} \mathrm{D}$ Salas, ${ }^{5} \mathrm{M} \mathrm{D}$ la vega, ${ }^{6} \mathrm{~A}$ Baroja, ${ }^{7} \mathrm{C}$ Natal, ${ }^{1} \mathrm{~F}$ Macià, ${ }^{8} \mathrm{M}$ Baré, ${ }^{9} \mathrm{~J}$ Galceran, ${ }^{10}$ I González-Román, ${ }^{1} \mathrm{X}$ Castells. ${ }^{1}$ Department of Epidemiology and Evaluation, IMIM-Parc de Salut Mar, CIBERESP, Barcelona, Spain; ${ }^{2}$ Galician breast cancer screening programme, Public health \& Planning Directorate, Health Office, Santiago de Compostela, Spain; ${ }^{3}$ Navarra Breast Cancer Screening Programme, Public Health Institute, CIBERESP, Pamplona, Spain; ${ }^{4}$ General Directorate Public Health \& Centre for Public Health Research, Valencia, Spain; ${ }^{5}$ General Directorate of Health Care Programmes, Canary Islands Health Service, Santa Cruz de Tenerife, Spain; ${ }^{6}$ La Rioja Breast Cancer Screening Programme. Fundacion Rioja Salud, Logroño, Spain; ' Unit of Analysis and Programs, Health Service of Asturias, Oviedo, Spain; ${ }^{8}$ Epidemiology and Assessment Unit UDIAT-Diagnostic Center, Corporació Parc Taulí, Sabadell, Spain; ${ }^{9}$ PDPCM, League Foundation and Cancer Prevention Research, IISPV, Reus, Spain; ${ }^{10}$ Castilla-Leon Breast Cancer Screening Programme, D.G. Salud Publica ID e I, SACYL, Valladolid, Spain

Introduction Adherence to breast cancer screening is affected by presence of previous false-positives and regular participation in previous invitations. Our aim was to estimate the long-term adherence to breast cancer screening and how false-positive and women's characteristics affect the probability of re-attending screening.

Methods Retrospective Cohort study of women aged 45-69 years invited to participate in any of 10 Spanish breast cancer screening programs between 1990 and 2006. Discrete time hazard models were used to estimate re-attendance probabilities, and to evaluate the effect of false-positives and women's characteristics on re-attendance.

Results We analysed information from 1371218 screened women who underwent 4545346 screening mammograms. The re-attendance probability was $81.7 \%$ (95\% CI 81.63 to 81.76$)$ at first screening, $88.1 \%$ (95\% CI 87.98 to 88.12$)$ at 3rd screening, and $95.6 \%(95 \%$ CI 95.52 to 95.73$)$ at 6 th screening. Women without a false-positive result were more likely to return to the following screening invitation. The re-attendance probability at first screening was $79.3 \%$ (99\% CI 79.0-79.6) and $85.3 \%$ (99\% CI 85.2-85.4) for women with and without a false-positive result, respectively. At sixth screening was $94.6 \%$ (99\% CI 93.8-95.4) and 96.0\% (99\% CI 95.8-96.1), respectively. The factors associated with a higher risk of failing to re-attend the following screening invitation were: age 65-69 years $(\mathrm{OR}=8.48$; $\mathrm{CI}$ 8.31-8.65), missing the first screening invitation $(\mathrm{OR}=1.12$; $\mathrm{CI} 1.11-1.14)$, and previous invasive procedures $(\mathrm{OR}=1.09$; CI 1.07-1.10).

Conclusion False-positive results and other women's characteristics affected the re-attendance to subsequent screening invitations. This information could be useful to provide the maximum available information to women invited to participate and improve compliance in subsequent screening invitations. 